\title{
Laparoscopic splenectomy in pregnancy - from contraindication to golden standard
}

\author{
Majesky $\mathrm{I}^{1}$, Daniel $\mathrm{I}^{1}$, Stefanikova $\mathrm{Z}^{2}$, Skultety $\mathrm{J}^{1}$, Koudelka $\mathrm{P}^{1}$, Hutan $\mathrm{M}^{1}$ \\ 2nd Clinic of Surgery, University Hospital Bratislava, Slovakia. imajesky@yahoo.com
}

\begin{abstract}
Laparoscopy has been holding its strong position within visceral surgery for decades. For several diagnoses, laparoscopy became the method of first choice. Laparoscopic splenectomy is a standardized procedure in patients indicated mostly due to haematological disorders. Apart from bleeding disorders, pregnancy used to be recognized as a contraindication to laparoscopic surgery. Splenic cysts are extremely rare during pregnancy; only seven cases have been described in literature. The authors had to deal with a patient treated and observed by haematologist for haemorrhagic splenic cyst and hypersplenism. Because of her low compliance, the patient did not undergo splenectomy in the past. Due to the gradual progression of disease and furthermore because of her pregnancy, the question of splenectomy became semi-urgent. The patient underwent laparoscopic splenectomy in second trimester, while not only the surgery alone, but also her early postoperative period and remaining pregnancy passed off uneventful. The authors suggest that even in pregnancy, the patients suffering from serious haematological disorders necessitating splenectomy could safely benefit from the advantages of laparoscopic approach, which also in these cases could become a gold standard (Fig. 2, Ref. 13). Full Text in PDF www.elis.sk.

Key words: laparoscopy, splenectomy, haemorhage, cyst, pregnancy.
\end{abstract}

The laparoscopic approach can be seen as one of milestones of surgery of the 20th century. Since the first laparoscopic appendectomy described in 1982 by German gynaecologist, Dr Semm, the laparoscopic approach has become popular among many surgeons. Owing to its well-known and unchallenged benefits, laparoscopy stands for a method of choice in some diagnoses, mostly accepted for symptomatic gall stones. Through the years of experience, more and more sophisticated procedures have been executed this way. Obviously, laparoscopic splenectomy has gained its own strong position. Nevertheless, there are several limits of laparoscopic approach, some from the patient and some from the surgeon alone. Contraindications to laparoscopy are relative and include an uncooperative patient, irreversible coagulation defects, severe congestive heart failure, respiratory insufficiency, suspected acute diffuse peritonitis, and the presence of distended bowel. If tense ascites is present, large-volume paracentesis can be performed as a preliminary step in laparoscopy. Previous laparotomy incisions may necessitate alteration of the usual trocar insertion site, or may represent a contraindication to the procedure. Most surgeons would not recommend laparoscopy in patients with preexisting disease conditions. Patients with cardiac diseases and COPD should not be considered good candidates for laparosco-

${ }^{1}$ 2nd Clinic of Surgery, University Hospital Bratislava, and ${ }^{2}$ Clinic of Haematology, University Hospital Bratislava, Bratislava, Slovakia

Address for correspondence: I. Majesky, MD, 2nd Clinic of Surgery, University Hospital Bratislava, Antolska 11, SK-851 07, Bratislava, Slovakia. Phone: +421.2.68673331 py. Laparoscopy may also be more difficult in patients who have had previous abdominal surgery as well as in patients with BMI over 35 . The elderly may also be at increased risk of complications of general anaesthesia combined with pneumoperitoneum. Laparoscopy does add to the surgical risk in patients with a lowered cardio-pulmonary reserve with regard to the consequences of pneumoperitoneum and prolonged operative time. In early times of laparoscopic era, pregnancy used to be recognized as an absolute contraindication $(1,2)$. Nowadays current estimates of nonobstetric surgery in pregnancy range from $0.1 \%$ to $0.2 \%$ $(3,4)$. Clearly the benefits of laparoscopic surgery for pregnant patients include less postoperative pain, earlier return to normal respiratory and gastrointestinal function, faster return to normal activity level and shorter hospital stay. Despite the advances in surgery, anaesthesiology and obstetrics, any surgical procedure in pregnancy could result in foetal loss from either spontaneous abortion or premature labour. In laparoscopy, the potential risks of anaesthesia are more important than the risks of laparoscopic surgery. There is a potential risk to the foetus due to trocar insertion, $\mathrm{CO}_{2}$ insufflation and technical limits of exposure due to enlarged uterus. Decreased uterine blood flow remains a question. Currently it is maintained that pneumoperitoneum may be safer than manual uterine retraction during open procedures. The risk for the mother is in accentuation of decreased venous return, decreased cardiac output, increased systemic and pulmonary vascular resistance, and increased blood pressure. Carbon dioxide insufflation to create pneumoperitoneum leads to decreased compliance of lungs and thoracic wall, diaphragm elevation and consecutive 
reduction in functional residual capacity of the lungs, increased peak airway pressure and increased partial pressure of arterial carbon dioxide (5). Nowadays, owing to broadening experience, laparoscopy is used to provide the benefits of minimally invasive approach also in disorders complicating the pregnancy. The most common laparoscopic nonobstetric procedures performed in pregnancy are laparoscopic appendectomy and laparoscopic cholecystectomy. Appendicitis occurs once in every 1,500-2,000 pregnancies, and the biliary tract diseases affect one in 1,600 to 10,000 pregnancies (6). Other procedures occur very occasionally, so does the laparoscopic splenectomy. The need for urgent splenectomy in pregnancy is rare, while the indications can include haemolytic crisis in hereditary spherocytosis, progressive growth of splenic cysts especially with hypersplenism and an arising risk of splenic rupture (7).

\section{Case presentation}

The study describes a case of a 25-year old woman, for 5 years observed and treated for a splenic cyst by haematologist. She had no history of serious cardiovascular or pulmonary disease. She has not undergone any surgery. Her history was free of any previous abdominal trauma. The serology for hydatid IgG was negative. Her BMI before she became pregnant was 20.57. Due to her lack of compliance, the patient formerly refused surgery indicated by haematologist. There was also a history of drug and alcohol abuse. For at least three years she did not visit any doctor. She became pregnant, and because of the progressive signs of hypersplenism and fast enlargement of the spleen, especially of its cystic portion, she decided to visit her haematologist again. Due to the progressive growth of spleen the patient felt uncomfortable due to intermittent dull pain in her left hypochondrium. The patient repeatedly underwent diagnostic procedures, including abdominal ultrasound as well as magnetic resonance imaging (Figs 1 and 2) a few weeks before surgery, thereby verifying the above-mentioned condition. After the informed consent had been

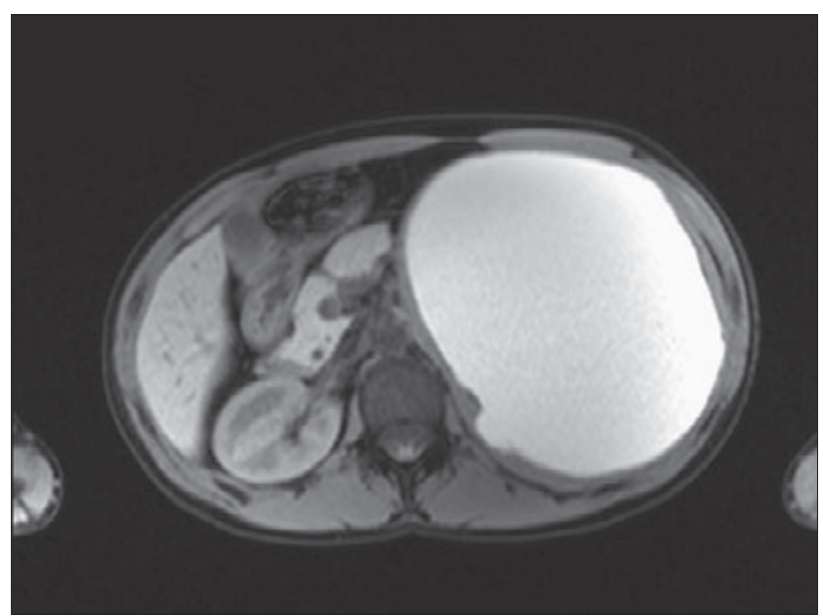

Fig. 1. Magnetic resonance imaging.

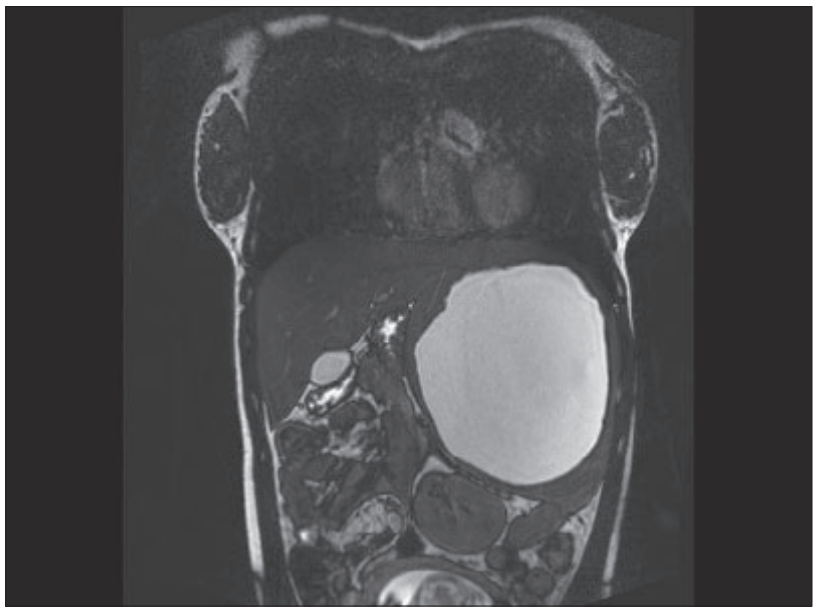

Fig. 2. Magnetic resonance imaging.

obtained, the patient was indicated for surgery in her second trimester. She underwent laparoscopic splenectomy. For VTE prevention we used LMWH, adjusted in the postoperative period according to laboratory results. The patient was positioned on her right flank, bent in the waist, with left arm abducted. We used four trocar approaches with special care to the enlarged uterus. The trocars were placed along a linear curve situated below the left costal margin from the midclavicular to the posterior axillary line while the optical port was situated a little bit closer to the costal margin than it would have been done in a non-obstetric patient. We used a Veress needle, insufflated the abdomen up to the $12 \mathrm{mmHg}$ pressure and maintained that pressure during the whole procedure, while the capnoperitoneum was monitored by the surgeon and anesthesiologist. Due the huge cystic portion of the enlarged spleen we decided to puncture the cyst primarily. We have evacuated more than 2,300 $\mathrm{ml}$ of sanguineous fluid. Without this manoeuvre, it would not have been possible to perform any safe surgical procedure inside the peritoneal cavity. We used a combined posterior-anterior approach, divided the splenocolic ligament, and mobilized the splenic flexure of colon. Then after elevating gently the lower pole of the spleen we divided the inferior polar vessels. With the use of ultrasonic scissors we divided the gastrosplenic ligament and thereby opened the omental bursa. We divided the short gastric vessels. After that we dissected the splenorenal ligament. At this point, the hilar vessels were divided with the use of endoGIA device. The spleen was put into the EndoCatch $^{\mathrm{TM}}$ bag and extracted through a small extended trocar incision in the left hypochodnrium after forceps morcellation. After haemorrhage control, we spilled saline solution over the left subphrenic region and put the drainage there. The drainage was removed on second postoperative day and the patient was released on fourth postoperative day without the need for blood transfusion. The entire postoperative period remained uneventful. The patient was observed on outpatient basis by her haematologist and gynecologist who noticed a rapid improvement in her labarotory signs (anaemia, thrombocytopaenia) with a physiologic course of pregnancy and delivery. 


\section{Discussion}

After successful laparoscopic management of appendicitis and gallbladder disease in pregnancy, surgeons began exploring laparoscopy in the management of other surgical diseases that arise in pregnancy. The spleen is the most vascularised organ in the body and its parenchyma is fragile. These properties, iincluding the fact that splenectomy is often performed for bleeding disorders, increase the risk of uncontrollable haemorrhage during laparoscopy. Cysts of the spleen in pregnancy are very rare with only seven cases described in literature while six of them were treated surgically and one was managed conservatively by means of analgesia, antibiotics and percutaneous aspiration (8). Splenic cysts carry a risk of haemorrhage, infection and rupture. Splenic rupture during pregnancy is known as splenic emergency syndrome associated with haemorrhagic shock. There is an increased risk of foetal death and high maternal mortality rates have been reported, namely up to $70 \%$ (9). Not only the primary disorder, but also the size of the spleen can limit the laparoscopic approach. Surgical treatment of splenomegaly is associated with prolonged operative time, blood transfusion requirements, higher conversion rates and longer hospital stay. Hand-assisted approach could be sometimes used to avoid conversion, thereby maintaining some benefits of laparoscopic approach, such as less postoperative pain, faster recovery of gastrointestinal function and shorter hospital stay, when compared with open splenectomy (10). Due to enlarged uterus, there is restricted space inside the abdominal cavity. During pregnancy, the second trimester is the preferred period for timing the surgery. The course of the disease allowed us to wait for three weeks after gaining the informed consent. When possible, the surgical intervention should be postponed until the second trimester due the possibility of impairing the organogenesis during the first trimester. On the other hand, the third trimester brings about space limitations due to enlarged gravid uterus and an increased risk of premature labour. During procedure, the surgeon should avoid manipulating the uterus. Since pregnancy induces a hypercoagulable state and the enlarged uterus with pneumoperitoneum enhances a lower-extremity venous stasis, appropriate measures against venous thromboembolism should be undertaken. Optimal VTE prevention would include intermittent pneumatic compression with early postoperative ambulation and use of LMWH. We did not observe any serious side effects after short LMWH administration during pregnancy in perioperative period. The most favourable position for patient during pregnancy is left lateral recumbent position because of diminished pressure of enlarged uterus on vena cava. As opposed to that, the laparoscopic splenectomy patient was positioned in right lateral decubitus position while bent in the waist, which is enormously important for approaching the spleen safely. This is why vigilant monitoring of intraoperative $\mathrm{CO} 2$ and blood pressure is mandatory. Laparoscopic splenectomy could be performed using anterior, semilateral or lateral approach according to surgeon's preference, spleen size and need for concomitant procedures. Anterior or "supine" position used to be applied in early years of laparoscopic splenectomy. Today, this position is indicated in case of concomitant procedures that need to be un- dertaken. Another choice could be a semilateral approach, when the patient's left side is elevated up to $45^{\circ}$. With this approach, the position could be adjusted during procedure to an anterior or lateral one. In a lateral or right-decubitus position, the patient is positioned at a $90^{\circ}$ angle on the table where the viscera are pulled down medially by gravity. With this approach, some authors have reported a statistically significant reduction in operating time, transfusion requirements and length of hospital stay (11). Trocar insertion poses a risk of uterine injury but actually we do not insist on Hasson technique to create the pneumoperitoneum; this depends on surgeon's experience. Routinely we use 12 or $13 \mathrm{mmHg}$ insufflation pressure, although pressures of $10-15 \mathrm{mmHg}$ can be safely used during pregnancy. Actually, SAGES guidelines contain a recommendation to minimize pneumoperitoneum pressure (to 8-12 $\mathrm{mmHg}$ ) while not to exceed $15 \mathrm{mmHg}$ (12). We have used four trocars during the procedure in typical positions. There are a few reports on cases of single incision splenectomy in literature (13), however none in pregnancy. The punction of the cyst enabling to reduce the size of the spleen could be used because there was no diagnosis or suspicion of malignant disease. Another choice for size reduction is that of splenic artery clipping. The procedure itself did not differ from that performed on non-obstetric patients. The size of the spleen is one of the most notable limits of laparoscopic approach, thus precise preoperative diagnostic procedures are of great value. Abdominal ultrasound and magnetic resonance imaging are preferred methods during pregnancy, although there are some records that special protocols of advanced CT imaging could be used with radiation doses below detrimental levels. According to surgeon's preferences, a variety of clips, sutures, monopolarbipolar coagulation modes (hooks, dissectors), ultrasound scissors (harmonic scalpel) or LigaSure devices could be used to dissect tissues while achieving haemostasis. For retrieving the spleen, the use of extraction bag is recommended. In order to diminish the risk of postoperative splenosis, care should be taken, especially during morcellation, not to violate the integrity of bag used for specimen extraction. Some authors recommend blunt instruments (fingers, ring forceps) for morcellation to avoid tearing the extraction bag and subsequent cell spillage.

\section{Conclusions}

Depending on local conditions, history of surgery, cardiovascular and pulmonary condition of patient, BMI, and surgeon's experience, there could be a group of patients who are suitable for laparoscopic approach including splenectomy during pregnancy and can benefit safely from minimally invasive surgery. Laparoscopic splenectomy is considered the gold standard of treatment of haematological disorders of the spleen in adult and paediatric patients and pregnancy seems not to represent an exception to this norm. It is a safe and effective technique resulting in less postoperative pain, faster functional recovery, faster return to normal activity level and shorter hospital stay when compared to open splenectomy. Furthermore, the perioperative morbidity of the laparoscopic approach is significantly lower. 


\section{References}

1. Gadacz T R, Talamini M A. Traditional versus laparoscopic cholecystectomy. Am J Surg 1991; 161 (3): 336-338.

2. Fitzgibbons R. Questions and answers: laparoscopic cholecystectomy. J Amer Med Ass 1991; 266: 269.

3. Kort B, Katy VL, Watson WJ. The effect of nonobstetric operation during pegnancy. Surg Gynecol Obstet 1993; 177: 371-376.

4. Kammerer WS. Nonobstetric surgery in pregnancy. Med Clin North Am 1987; 71: 551-560.

5. Kucykowski KM. Laparoscopic procedures during pregnancy and the risks of anesthesia: what does the obstetrician nedd to know? Arch Gynecol Obstet 2007; 276: 201-209.

6. Alkis I, Kurdoglu M, Kurdoglu Z. Nonobstetric surgical intervention in pregnancy. Eastern J Med 2010; 15: 1-6.

7. Habermaly B, Sauerland S, Decker G et al. Laparoscopic splenectomy: the clinical practice guidelines of the European Association for Endoscopic Surgery (EAES). Surg Endosc 2008; 22: 821-848.
8. Mahran AM, Bodley R, Farouk M, Aasworth F. Conservative management of giant splenic cyst during pregnancy. Gynecol Surg 2010; 7: $49-51$.

9. Tanchev S, Papova M, Slavov I. The "splenic emergency syndrome" during pregnancy. A report of two cases. Akush Ginekol 1992; 31: 32-34.

10. Kusminsky RE, Boland JP, Tiley EH, Deluca JA. Hand-assisted laparoscopic splenectomy. Surg Laparosc Endosc 1995; 5: 463-467.

11. Gossot D. Laparoscopic splenectomy: value of the posterior approach. Ann Chir 1998; 52: 940-945.

12. Guidelines for laparoscopic surgery during pregnancy. Surg Endosc 1998; 12: 189-190.

13. Barbaros U, Dinccag A. Single incision laparoscopic splenectomy: the first two cases. J Gastrointest Surg 2009; 13: 1520-1523.

Received August 24, 2011. Accepted January 23, 2013. 